\title{
Social Network Analysis across Healthcare Entities, Orange County, FL, 2016
}

\author{
Danielle Rankin* \\ Epidemiology, Florida Department of Health in Orange County, Orlando, FL, USA
}

\section{Objective}

To create a baseline social network analysis to assess connectivity of healthcare entities through patient movement in Orange County, Florida.

\section{Introduction}

In the realm of public health, there has been an increasing trend in exploration of social network analyses (SNAs). SNAs are methodological and theoretical tools that describe the connections of people, partnerships, disease transmission, the interorganizational structure of health systems, the role of social support, and social capital $^{1}$. The Florida Department of Health in Orange County (DOHOrange) developed a reproducible baseline social network analysis of patient movement across healthcare entities to gain a county-wide perspective of all actors and influences in our healthcare system. The recognition of the role each healthcare entity contributes to Orange County, Florida can assist DOH-Orange in developing facility-specific implementations such as increased usage of personal protective equipment, environmental assessments, and enhanced surveillance.

\section{Methods}

DOH-Orange received Centers for Medicare and Medicaid Services data from the Centers for Disease Control and Prevention Division of Health Care Quality Promotion. The dataset contains the frequency of patients transferred across Medicare accepting healthcare entities during 2016. We constructed a directional sociogram using R package statnet version 2016.9, built under R version 3.3.3. Node colors are categorized by the type of healthcare entity represented (e.g., longterm care facilities, acute care hospitals, post-acute care hospitals, and other) and depict the frequency of patients transferred with weighted edges. Node sizes are proportional to the log reduction of the total degree of patients transferred, and are arranged with the FruchtermanReingold layout. We calculated standard network indices to assess the magnitude of connectedness across healthcare entities in Orange County, Florida. Additionally, we calculated node-level indices to gain a perspective of the strength of each individual entity.

\section{Results}

A total of 48 healthcare entities were included in the sociogram, with $44 \%$ representing Orange County, Florida. Although the majority of the healthcare entities are located in nearby counties, $90 \%$ of patient movement occurred across Orange County entities. The range of patient movement was 1 to 5196 with a median of 15 patients transferred in 2016. The network in Orange County is sparse with a density of 0.05 , but the movement of patients across the healthcare entities is predominately symmetric (reciprocity $=97 \%$ ). The sociogram is centralized (degree centrality $=0.70$ ) and contains a vast amount of entities that serve as connectors (betweenness centrality $=0.53$ ). The node-level indices identified our acute care hospitals and long term acute care hospitals are the connectors of our county health system.

\section{Conclusions}

The SNA of patient movement across healthcare entities in Orange County, Florida provides public health with knowledge of the influences entities contribute to the county healthcare system.
This will contribute to identifying changes in the network in future research on the transmission risks of specific diseases/conditions, which will enhance prioritization of targeted interventions within healthcare entities. In addition, SNAs can assist in targeting disease control efforts during outbreak investigations and support health communication. A SNA toolkit will be distributed to other local county health departments for reproduction to determine baseline data and integrate county-specific SNAs.

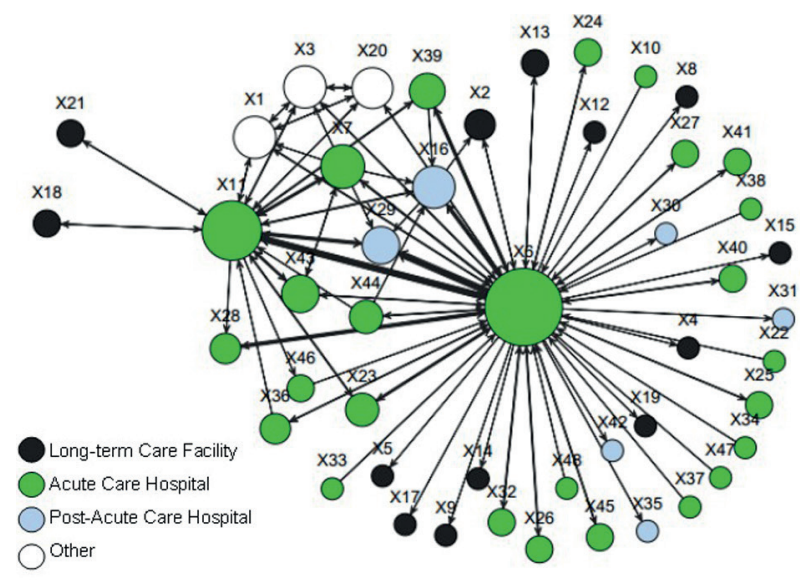

Note: each node ( $\mathrm{O}$-circie) indicates a separate healthcare entify; node sizes ( $\mathrm{O}$-circle) depict the total degree* (indegree+outdegree) 0.3 ; edges (—_-lines) are weighted with a log reduction (divided by 50 ) of the volume of patients shared from facility to facility, arrows $(\longrightarrow)$ denote the direction of patients being shared across healthcare entities; length of lines depict the speed of patient movement

tDefinition of fotal degree: the overall volume of connections that exist to each specific healthcare entity

\section{Keywords}

sna; social network analysis; patient movement

\section{Acknowledgments}

The Florida Department of Health in Orange County would like to thank Sarah Matthews for assistance with obtaining the data to conduct the social network analysis. Additonally, we would like to express our gratitude to The Centers for Disease Control and Prevention (CDC) Division of Healthcare Quality Promotion for providing the dataset used in the analysis of this study. I would like to express my gratitude to Dr. German Gonzalez, Alvina Chu, and Karen Elliott from the Florida Department of Health for mentoring and guiding me through my fellowship.

\section{References}

1. Luke DA, Harris JK. Network Analysis in Public Health: History, Methods, and Applications. Annual Review of Public Health. 2007;28(1):69-93. doi:10.1146/annurev. publhealth.28.021406.144132.

\section{*Danielle Rankin}

E-mail: danielle.rankin@flhealth.gov 